\title{
Sistema de ejercicios profilácticos para la prevención de las lesiones de rodilla en el Karate Do System of prophylactic exercises for the prevention of knee injuries in Karate Do \\ *Aracely Moraima Cabeza Toro, **Lisbet Guillen Pereira, ***Manuel Gutierrez Cruz, **Lidys Padrón Fernández, ***Danilo Fernando Vargas Vera, ***Ricardo Manuel Ortega Oyarvide \\ *Universidad Estatal de Milagro (Ecuador), ${ }^{* *}$ Universidad Metropolitana del Ecuador (Ecuador) **Universidad Central del Ecuador (Ecuador), ${ }^{* * *}$ Universidad Estatal de Guayaquil (Ecuador)
}

Resumen: La investigación se centró en diseñar un sistema de ejercicios profilácticos para prevenir las lesiones de rodilla, para ello se transitó por una fase diagnostica empleando cuatro profesores de karate de la Federación de los Ríos y 20 karatecas de la categoría 14-16 años, la recopilación de la información estuvo respaldada por un análisis estructural funcional de la rodilla, revisión de documentos, la observación y la entrevista, los resultados permitieron la elaboración de la propuesta la cual fue sometida a la evaluación de 15 expertos, posteriormente se procedió a la fase de validación empírica para lo cual se empleó un pre-experimento pedagógico donde intervinieron las 14 principales figuras del equipo, el proceso estuvo respaldado por una hipótesis de investigación, siguiendo la lógica de evaluación (pre_test), intervención-despliegue y evaluación (post_test) de la amplitud articular en la flexión de la articulación de la rodilla y de la fuerza máxima de los músculos que inervan la articulación: cuádriceps, bíceps femoral y gemelos. Para la contrastación del pre_test con el post_test fue necesario determinar la normalidad de los datos, se empleó un Test de Bondad de ajuste: Kolmogorov-Smirnov: KS, lo que permitió la aplicación de la prueba T para diferencias de medias, la significación de cambios fue de p=0.001 comprobando Hi en concordancia es posible plantear que un sistema de ejercicios específicos elaborados a partir del principio de estructura y función, garantiza la prevención de lesiones de rodillas asociadas las exigencias físicas que se deriva de la ejecución de la kata y el kumité.

Palabras claves: Prevención de lesiones, sistema de ejercicios profilácticos, Karate, articulación de la rodilla, Goniometría.

Summary: This research focused on designing a system of prophylactic exercises to prevent knee injuries. A diagnostic evaluation employed four karate teachers from the Federation of Rivers and 20 karate athletes from the 14-16-year-old category. Data collection was supported by a functional structural analysis of the knee, document review, as well as observations and interviews. The results allowed for the elaboration of a proposal submitted to the evaluation of 15 experts. Following that, the phase of empirical validation started, for which a pedagogical pre-experiment was implemented with the participation of the 14 most prominent figures of a team. The process was supported by a research hypothesis, following the logic of evaluation (pre_test), intervention-implemetation, and evaluation (post_test) of the joint range during the flexion of the knee joint and the maximum strength of the muscles surrounding it: quadriceps, hamstrings, and calves. For pre_test/post_test assessment, the normality of data needed to be determined. Therefore, the KolmogorovSmirnov Goodness Test of Adjustment was used (KS), which confirmed that $\mathrm{T}$ test for differences of means could be employed. T tests results showed a significance of changes of $p=0.001$, corroborating the Hi. In conclusion, we can assume that a system of specific exercises developed from the principle of structure and function guarantees the prevention of knee injuries associated with the physical demands resulting from the execution of the kata and the Kumite.

Keywords: Prevention of injuries, system of prophylactic exercises, Karate, knee joint, Goniometry.

\section{Introducción}

En el ámbito deportivo el Karate Do es uno de los deportes de combate más conocido a nivel mundial, se caracteriza por el enfrentamiento cuerpo a cuerpo y su fin fundamental es vencer al oponente utilizando técnicas de ataque y defensa.

Su desarrollo a nivel mundial ha sido paulatino y significativo, muestra de ello es que a partir del año 1993 el Karate Do se incluye en los Juegos regionales y continentales del ciclo olímpico, en el 1998 el Comité Olímpico Internacional (COI), reconoce al Karate como deporte Olímpico y en el 2016 se incluye en los Juegos Olímpico de Tokio 2020 (Mundo Deportivo, 2016).

Lo antes expuesto ha redimensionado el enfoque de la preparación de los atletas del karate a nivel internacional, como consecuencia se ha incrementado las exigencias en función del rendimiento deportivo y el reordenamiento del sistema de preparación buscando cambios más profundos en el orden fisiológico y morfológico, mediante el empleo de cargas cada vez más concentradas.

Fecha recepción: 13-02-19. Fecha de aceptación: 31-05-19 Lisbet Guillen Pereira guillenp7212@gmail.com
En este orden la periodización se asume como el enfoque organizado del sistema de preparación del atleta que implica ciclos progresivos de entrenamiento dentro y fuera de Jogay, como parte de un período específico inherente al Ciclo Olímpico para el cual se está trabajando.

En concordancia la periodización del entrenamiento constituye un soporte de referencia básico para establecer un proceso de prevención de lesiones de los atletas que son sometidos a altas cargas con un componente significativo de variación regular y sistemática de las mismas, de manera que puedan cumplir sin dificultad el proceso de preparación establecido a largo plazo (Fernández, 2014; Fernández \& Busto, 2015; Álvarez \& Murillo, 2016; De Dios, 2016).

En tal sentido García (2012) acota que la lesión deportiva representa el factor más limitante de los deportistas, cuya problemática le ha llamado la atención a los profesionales del deporte, los cuales reconocen en estas la necesidad de mejorar las medidas preventivas y reducir el número de lesiones, para poder disponer de sus deportistas el mayor tiempo posible y no amenazar su rendimiento (García, 2012; Adalid, 2014; Prieto, 2015; Penichet-Tomás, Ortega, Jiménez-Olmedo, Pueo, \& Espina, 2019).

Visto desde esta perspectiva resulta necesario comprender la implicación de todo el sistema ostiomioarticular del 
atleta en la ejecución de las acciones, para manejar la dimensión preventiva de la preparación. Su desconocimiento expone a los atletas a lesiones recurrentes que desvirtúan el rendimiento deportivo.

Según Pitarch (2016) en el Karate pueden aparecer tanto lesiones crónicas como agudas, en tal sentido las primeras aparecen en un momento específico del proceso de preparación o en la propia dinámica de competición como resultado de la exposición de altas cargas, como consecuencia del proceso de enfrentamiento directo o el impacto traumático que se deriva de golpes propinados y las segundas se asocian al desgaste producido por la práctica de la disciplina o la inadecuada curación de una lesión crónica.

Para Villaquirán, Portilla-Dorado, Vernaza-Pinzón (2016) las lesiones agudas muestran una importante carga a nivel tisular ocasionando una deformación súbita e irreversible del tejido lo cual aparece como resultado de caídas o golpes directos.

En este punto Pitarch (2016) precisa que dentro de las lesiones agudas más recurrentes que se registran en los karatecas sobresalen las siguientes:

- Lesiones en el pulgar: estas pueden aparecer en las practicantes del área formativa, especialización o del alto rendimiento al colocar de forma incorrecta el pulgar de la mano cuando se cierra el puño.

- Contracturas musculares: ocasionadas por movimientos bruscos, muestran una alta presencia de dolor y es palpable al tacto.

- Desgarro muscular: se presentan como resultado de los movimientos bruscos y rápidos que se realizan, produce un dolor fuerte e intenso o aparición de un hematoma combinado con la imposibilidad de mover el músculo afectado.

- Esguince de tobillo: en esta lesión es preciso considerara que en la modalidad de Kumite es la articulación más expuesta, como resultado de saltos continuos de carácter variables.

- Hemorragia nasal: se origina como resultado de la ruptura de una o varias de las venas que se encuentran muy superficialmente situadas bajo la mucosa que «tapiza» las fosas nasales.

- Dolores Abdominales: se presentan como resultado de golpes fuertes a la zona, ocasionando mayor trauma con la musculatura abdominal relajada.

- Traumatismo en la cabeza y en la zona cervical: aparecen como resultado de los golpes dirigidos a las zonas, es preciso destacar que este tipo de acción está limitada y penalizada, apreciada por los árbitros como golpe excesivo.

En relación a las lesiones crónicas Villaquirán et al (2016) sostienen que estas se generan como consecuencia de una sobrecarga repetitiva, como resultado de las exigencias físicas y la exposición del segmento afectado a lo largo del tiempo, en tal caso estas aparecen cuando la lesión logra exceder el umbral de daño tisular, ante el exceso de actividad física, la aplicación de cargas exageradas o un inadecuado manejo de la recuperación o correlación trabajo descanso.

Al referirse al tema Pitarch (2016) define las siguientes lesiones crónicas como las más recurrentes en la práctica del Karate Do:

$$
\begin{aligned}
& \text { - Esguinces crónicos de tobillo } \\
& \text { - Lumbalgias }
\end{aligned}
$$

\section{- Lesiones del ligamento cruzado anterior \\ - Lesiones de rodilla}

$\mathrm{Al}$ respecto resulta necesario acotar que en la dinámica del combate se produce en las extremidades inferiores una sobrecarga de las estructuras anatómicas, como resultado de los continuos saltos y desplazamientos, ello incrementa el riesgo de aparición de lesiones musculares y articulares, esta última mayormente a nivel de rodilla (Miyamoto, Bosco \& Sherman, 2009).

En este orden diferentes autores (López-Liria, VegaRamirez, López-Liria, Catalán-Matamoros, Padilla-Góngora, Martínez-Cortés \& Mesa-Ruiz, 2012; Feria, De Hoyo, Fernández, Romero, Cortés \& Sañudo, 2014a; Sánchez, Rodríguez-Mansilla \& González, 2015; Romero-Moraleda, Cuéllar, González, Bastida, Echarri, Gallardo \& Paredes, 2017) reconocen las lesiones en la rodilla como una de las más recurrentes y de lenta recuperación.

Al referirse a las repercusiones clínicas de las lesiones en la rodilla Ayala-Mejías, García-Estrada \& Alcocer (2014) apuntan que la mayor incidencia de lesiones del ligamento cruzado anterior se produce como consecuencia de traumatismos indirectos sobre la rodilla durante la práctica deportiva, las cuales frecuentemente no son diagnosticadas en el momento inicial. Afirman que los síntomas más frecuentes tras la lesión son dolor, tumefacción articular leve y sensación de fallo-inestabilidad de rodilla, fundamentalmente en actividades de torsión-recorte-desaceleración.

A pesar de la recurrencia de la presencia de lesiones como resultado de la práctica deportiva aun se ha constatado importantes limitaciones en relación a los procedimientos que garantizan la efectividad de la prevención de lesiones y el sistema de contenido que se elige para asegurar que estas no se presenten en la dinámica de competición.

Al respecto se reconocen importantes vacíos teóricos que desvirtúan la calidad de la prevención, en concordancia los ejercicios seleccionados no se alinean a ciertos aspectos que clarifican y fundamentan la necesidad de su utilización (Feria, et al., 2014a; Latorre, Cámara \& Pantoja, 2014).

En virtud de lo expresado se considera que el dominio de las características del deporte y su implicación anatomofisiológica en la ejecución de las acciones clarifica la necesidad de fortalecer planos musculares específicos que se comprometen directa e indirectamente en la calidad de la ejecución y la efectividad de la acción.

Contradictoriamente el incremento de las exigencias del sistema de competición en el Karate tanto para la modalidad de Kata (combate imaginario contra uno o varios adversarios) como para el Kumite (trabajo de enfrentamiento directo), ha traído consigo el aumento de la presencia de lesiones, lo cual ha llamado la atención de los profesionales del área.

En este orden el proceso de prevención que se impone ante las exigencias del sistema de preparación, desarrollo físico, técnico y táctico del Karate no permite desconocer los músculos y articulaciones con una implicación determinante o condicionante para la acción deportiva, así como la cadena biocinemática implicada para su fortalecimiento con un enfoque preventivo.

El Ecuador ha tenido la oportunidad de alcanzar grandes logros en esta disciplina deportiva, obteniendo resultados a nivel regional, centroamericano y panamericano, sin embar- 
go, la presencia de lesiones ha causado limitaciones en la participación en torneos de importancia.

\section{Metodología}

En el presente estudio se utilizó una investigación experimental donde se parte de un análisis descriptivo del estado del objeto de estudio, elaboración de la alternativa, validación teórica de la propuesta que da tránsito a la fase experimental la cual se ancla en la validación empírica de la propuesta.

En virtud de lo expresado la investigación transitó por tres fases: Diagnóstico, elaboración y validación, esta última estuvo conformada por dos momentos, uno centrado en la validación teórica de la propuesta por parte de los expertos seleccionados, y la segunda se centró en la validación empírica, en este punto la lógica de la investigación en el preexperimento transitó por tres momento:

Experimento (Pre-Experimento pedagógico)

1. Pre-test: Evaluación de dos dimensiones que develan el estado de las capacidades: amplitud articular y fuerza máxima de los músculos que están asociados a las exigencias fiscas de la rodilla

a) Prueba de goniometría de la articulación de la rodilla: Permite determinar la amplitud articular durante la flexión de la articulación de la rodilla (Goniometría de la articulación de la rodilla)

b) Prueba de Fuerza máxima (Cuádriceps, Bíceps femoral, Gemelos/Tobillo)

\section{Intervención pedagógica:}

a) Taller de preparación metodológicas de los profesores para el despliegue de la propuesta en la práctica

b) Despliegue de la propuesta por un espacio de 19 semanas (periodo comprendido entre el dos de septiembre del 2018 al 11 de enero del 2019)

3. Post-test: Evaluación de las dos dimensiones bajo las mismas condiciones que en el primer momento

\section{Población y muestra}

Para dar respuesta a los objetivos planteados se trabajó en la fase diagnóstica con cuatro entrenadores de Karate Do de la categoría Juvenil de la Federación Deportiva de los Ríos, los que representan el 100\% de los profesores que son responsables de la preparación de la categoría objeto de estudio. Intervienen además los 20 karatecas juveniles del equipo, los cuales representan el $100 \%$ de la población que conforma la categoría juvenil de 14-16 años.

Se tomó como referente a cuatro atletas que fueron objeto de lesión en la articulación de la rodilla, los que aportaron información valiosa para el diagnóstico del objeto de estudio.

Para evaluar la calidad formal de la propuesta se procedió a definir un conjunto de expertos de un total de 36.

La selección e intervención de los expertos siguió la lógica de tres etapas:

Gráfico 1. Etapas del proceso de selección de los expertos
}

En la fase de validación empírica de la propuesta se empleó a los 14 atletas que clasifican como principales figuras del equipo Juvenil de karate de la categoría 14-16 años, los cuales representan el 70\% de la población, seleccionados mediante un muestro no probabilístico intencional, de la esta muestra cuatro son mujeres y 10 son hombres.

El proceso de entrenamiento de los karatekas se distingue por su carácter multidisciplinar, así es que para hacer un estudio objetivo del mismo la deberá partirse de un análisis, que permitan distinguir los procesos que se relacionan, sin desestimar sus modos de relación, es a lo que podría llamarse operacionalización de la variable que subyace en este complicado proceso

\section{Métodos y/o técnicas}

En concordancia en el cumplimiento de los objetivos se utilizaron diferentes métodos del nivel teórico, empírico, estadístico matemático y técnicas que permitieron la recogida y procesamiento de la información dentro de los que se encuentran el método:

Analítico-Sintético. Método Hipotético. Inductivo-Deductivo, la Modelación: la cual permitió modelar la dinámica del flujo de la estructura procesual mediante una entrada, un proceso y una salida, establecer, el método Sistémico estructural Funcional, como método del nivel empírico dio la posibilidad de articular cada uno de los elementos y componentes del sistema, engranar cada grupo de ejercicio de manera que tributara al objetivo general de la propuesta y establecer un proceso de retroalimentación mediante el sistema de evaluación.

En este orden la Revisión de documentos facilitó la evaluación de la información sobre las especificidades de los documentos que norman la preparación de los karatekas en la Federación Provincial de los Ríos en Ecuador, la fuente, actualidad y relevancia de los mismos. La Observación científica permitió evaluar la actuación de los profesores durante el entrenamiento de las acciones relacionadas con la función de la rodilla, en concordancia se evaluó la selección y utilización de medios específicos y auxiliares, además de los criterios de dosificación y los modos de implementación de la relación trabajo descanso.

Como técnica la entrevista permitió acopiar información mediante la conversación profesional centrando la atención en la valoración del carácter profiláctico del proceso de preparación aplicado; de manera que permitió identificar el nivel de satisfacción de los atletas y la presencia de experiencia de lesiones, en correspondencia participaron los cuatro profesores que forman parte del equipo de preparación de la categoría y los 20 atletas que conforman el equipo juvenil 14-16 años de Karate de Federíos. En virtud de lo expresado la triangulación metodológica permitió contrastar la información desde diferentes ángulos para arribar a las conclusiones parciales

Se empleó el experimentos que como método permitió el despliegue de la validación empírica y la comprobación de la hipótesis de investigación planteada, dada las características del escenario y de la muestra objeto de estudio se utiliza un pre-experimento no participativo, desarrollado en condiciones naturales.

Se empleó la medición, la cual dio la posibilidad de com- 
probar en dos momentos la amplitud del arco articular en la flexión de la articulación de la rodilla en cada pierna, de igual manera se obtuvo información cuantitativa en relación a la fuerza máxima de los músculos asociados a los pateos y desplazamientos que comprometen la articulación de la rodilla de los karatekas de la categoría juvenil de 14-16 años de Federíos y que determinan el desempeño de los karatekas tanto en kata como en kumité. En este punto el empleo de la prueba se utilizó para la evaluación de la amplitud del arco articular de la rodilla y de la fuerza máxima en los segmentos comprometidos en la preservación de la rodilla durante el desempeño del atleta. Para proceder a la contrastación del pre test y el pos-test fue necesario la determinación de la normalidad de los datos cuantitativos por lo que se empleó un Test de Bondad de ajuste: Kolmogorov-Smirnov: KS, lo que dio paso a la aplicación de la prueba $\mathrm{T}$ para diferencias de medias en cada una de las pruebas, para ello se definió la siguiente hipótesis de investigación.

$\mathrm{H}_{i}$ : Un sistema de ejercicios específicos elaborados a partir del principio de estructura y función, garantiza la prevención de lesiones de rodillas asociadas las exigencias físicas que se derivan de la ejecución de la kata y el kumité en atletas juveniles de la categoría 14-16 años de la Federación Deportiva de los Ríos.

Esta hipótesis estuvo respaldada en las siguientes hipótesis estadísticas, definidas para la contrastación de los resultados de cada una de las pruebas realizadas en la práctica:

1. Prueba de Goniometría en el pie izquierdo

$\mathrm{H}_{0}$ : No existen diferencias significativas entre el Pre_test y Post_test respecto a los resultados de la prueba de Goniometría en el pie izquierdo

$\mathrm{H}_{1}$ : Sí existen diferencias significativas entre el Pre_test y Post_test respecto a los resultados de la prueba de Goniometría en el pie izquierdo

2. Prueba de Goniometría en el pie derecho

$\mathrm{H}_{0}$ : No existen diferencias significativas entre el Pre_test y Post_test respecto a los resultados de la prueba de Goniometría en el pie derecho

$\mathrm{H}_{1}$ : Sí existen diferencias significativas entre el Pre_test y Post_test respecto a los resultados de la prueba de Goniometría en el pie derecho

3. Prueba de fuerza máxima de Cuádriceps

$\mathrm{H}_{0}$ : No existen diferencias significativas entre el Pre_test y Post_test respecto a los resultados de la prueba de Cuádriceps

$\mathrm{H}_{1}$ : Sí existen diferencias significativas entre el Pre_test y Post_test respecto a los resultados de la prueba de Cuádriceps

4. Prueba de fuerza máxima de Bíceps Femoral

$\mathrm{H}_{0}$ : No existen diferencias significativas entre el Pre_test

y Post_test respecto a los resultados de la prueba de Bíceps Femoral

$\mathrm{H}_{1}$ : Sí existen diferencias significativas entre el Pre_test y Post_test respecto a los resultados de la prueba de Bíceps Femoral

Se asume como valor prefijado .05 para el rechazo de las hipótesis nulas.

\section{Presentación de la propuesta}

La rodilla es una de las articulaciones más complejas del sistema ostiomioarticular del hombre, en ella también se insertan ciertos músculos muy potentes que si bien ayudan a la eficacia de sus funciones, también pueden resultar muy nocivos si la preparación entre ellos no ha sido integral y equilibrada.

Según De Arma, Lorente \& Perdomo (2001) el sistema como aporte práctico tiene un carácter analítico y muestra al objeto en su dimensión estructural, en este se modifica su aspecto estático (estructura, componentes, organización y relaciones funcionales). Por tanto la propuesta que se presente, acorde con lo planteado por los autores, estructuralmente pretende enfatizar en la reorganización de los componentes y relaciones del objeto, a partir de los principios de estructura y función.

En tal sentido se plantea que un sistema estructuralmente podría contener los siguientes elementos:

- Marco epistemológico: Fundamentación y justificación de su necesidad

- Contexto social en el que se inserta el sistema

- Representación gráfica

- Explicación de la significados, exigencias, criterio de uso, argumentación sobre sus cualidades

- Formas de instrumentación: Recomendaciones, alternativas variantes

- Evaluación

Atendiendo a lo antes expuesto la propuesta quedó estructurada de la siguiente forma:

Objetivo general, sistema de objetivos específicos, Sustento teórica del sistema, Representación gráfica, sistema de ejercicios, sistema de evaluación, Orientaciones metodológicas generales para su implementación.

\section{Representación gráfica del sistema}

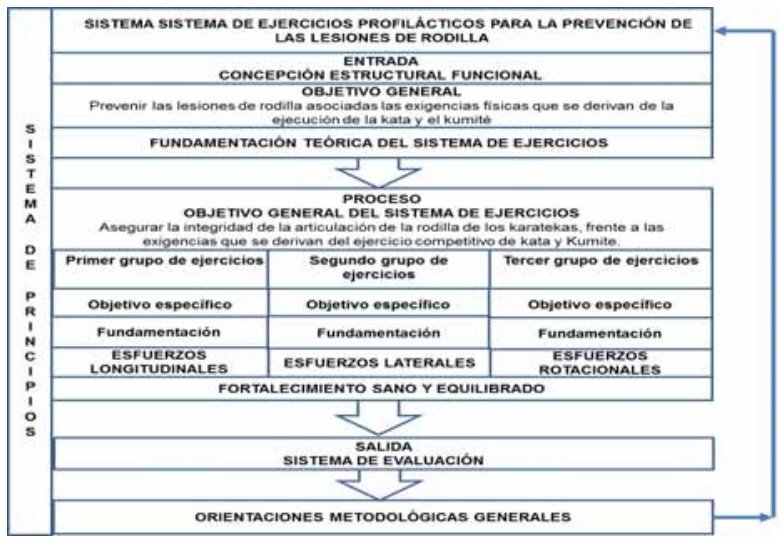

Gráfico 2: Representación esquemática del sistema de ejercicios

Objetivo general del sistema de ejercicios: Asegurar la integridad de la articulación de la rodilla de los karatekas, frente a las exigencias que se derivan del ejercicio competitivo de kata y Kumite.

Primer grupo de ejercicios

Objetivo específico: Fortalecer los grupos musculares comprometidos con la flexión y extensión de la pierna, así como las estructuras orgánicas de la articulación de la rodilla.

\section{Fundamentación}

Este grupo de ejercicios incluye aquellos grupos muscu- 
lares que están relacionados con la flexión y extensión de las piernas, que son las formas más naturales de movimientos de la articulación de las rodillas, creando así las condiciones para la ejecución de otras acciones más especializadas y de mayor exigencia. Por lo tanto, además de contribuir al fortalecimiento de estas estructuras orgánicas, también sirven de entrada al proceso de entrenamiento.

\begin{tabular}{|c|c|c|c|}
\hline Número & Descriן & ción del ejercicio & Aspectos procedimentales \\
\hline $1^{\circ}$. & \multirow{5}{*}{$\begin{array}{l}\text { Con } \\
\text { - apoyo } \\
\text { plantar }\end{array}$} & $\begin{array}{l}\text { Cuclillas con un apoyo sin sobre } \\
\text { peso }\end{array}$ & \multirow{7}{*}{$\begin{array}{l}\text { El trabajo a realizar debe } \\
\text { - comenzar por ejercicios con } \\
\text { - ambos apoyos de las piernas, } \\
\text { primero sin sobrepeso y luego } \\
\text { - con sobrepeso; en cualquiera de } \\
\text { los casos ayudan a consolidar la } \\
\text { - asimilación de la postura. } \\
\text { El aumento del peso y la } \\
\text { complejidad del ejercicio son } \\
\text { procedimientos eficaces para ser } \\
\text { consecuente con el principio del } \\
\text { carácter creciente y variable de } \\
\text { la carga de entrenamiento. } \\
\text { No debe subestimarse el } \\
\text { entrenamiento del bíceps } \\
\text { femoral que tiene } \\
\text { principalmente una función } \\
\text { compensatoria. }\end{array}$} \\
\hline $2^{\circ}$. & & Cuclillas con dos apoyos y sobrepeso & \\
\hline $3^{\circ}$. & & $\begin{array}{l}\text { Asalto al frente con sobrepeso } \\
\text { (tijeras) }\end{array}$ & \\
\hline $4^{\circ}$. & & $\begin{array}{l}\text { Extensión de tobillos sentado con } \\
\text { sobre peso }\end{array}$ & \\
\hline $5^{\circ}$. & & $\begin{array}{l}\text { Asalto sobre apoyo alto con } \\
\text { elevación de pierna de pivot y } \\
\text { extensión del tobillo }\end{array}$ & \\
\hline $6^{\circ}$. & & $\begin{array}{l}\text { Curl de piernas acostado. } \\
\text { (bíceps femoral) }\end{array}$ & \\
\hline $7^{\circ}$. & $\begin{array}{l}\text { Sin } \\
\text { apoyo } \\
\text { plantar }\end{array}$ & $\begin{array}{l}\text { Extensión de piernas sentado y con } \\
\text { sobrepeso (cuádriceps femoral) }\end{array}$ & \\
\hline
\end{tabular}

\section{Segundo grupo de ejercicios}

Objetivo específico: Fortalecer las estructuras orgánicas laterales internas y externas de, con vistas a afrontar aquellos esfuerzos técnicos que no son totalmente congruente con la disposición anatómica de la rodilla.

\section{Fundamentación}

Si el karate es un deporte de esfuerzo variable a partir de acciones cuyas estructuras se caracterizan por ser acíclicas variadas, entonces el entrenamiento de la rodilla no debe ser en una sola dirección, aun cuando se trate de una articulación mono axial. De ahí la importancia de fortalecer la musculatura que le es afín en otras direcciones, de modo que se pueda soportar con éxito los cambios bruscos de dirección, las aceleraciones y desaceleraciones tan frecuentes en este deporte.

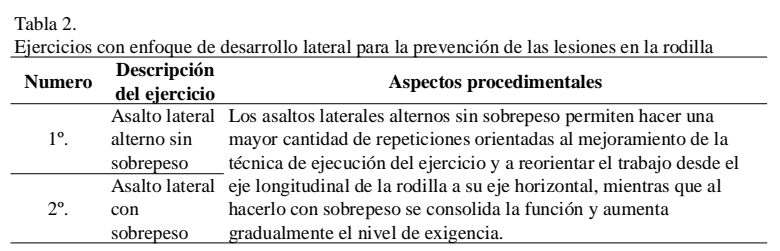

\section{Tercer grupo de ejercicios}

Objetivo específico: Fortalecer las estructuras osteomusculares que garantizan la eficacia de la ejecución de los pateos circulares y al frente.

\section{Fundamentación}

Los ataques y defensas a partir mediante el uso de pateos, derribos o barridos son muy frecuentes, lo mismo que las torsiones de las caderas y las rotaciones de los apoyos durante la ejecución de las katas y kumite, por lo tanto el sobreuso de estas acciones unido a que sus estructuras no responden directamente a la condición de la rodilla, puede convertirse en causa de lesiones no deseadas. Esta razón justifica la necesidad de los ejercicios que a continuación se relacionan.

Por otra parte, el fortalecimiento de la musculatura comprometida con los movimientos de los ejes longitudinal y horizontal, desestimando el trabajo en el eje sagital, puede conducir a una descompensación y falta de equilibrio en la preparación, que también es conducente a lesiones.

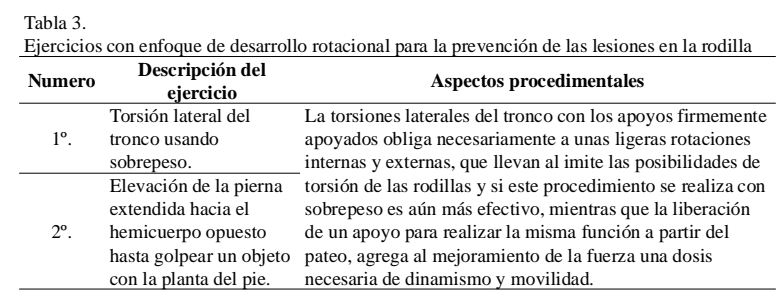

\section{Sistema de evaluación del sistema de ejercicio}

Para comprobar la eficacia del sistema de ejercicios creado se diseñó un instrumento que considera tres parámetros, los cuales están asociados a los componentes del sistema, y resultado de la investigación; estos dan respuesta fundamentalmente a las exigencias de las posturas y desplazamientos tanto en kata como en kumité, así como a las acciones de pateo en cualquiera de las direcciones, en concordancia se mide: amplitud angular durante la flexión de la articulación de la rodilla y fuerza muscular asociada a los pateos y desplazamientos:

\section{Nombre de la Prueba 1: Flexibilidad en la articulación de la rodilla}

Objetivo: Evaluar la amplitud angular que se deriva de la flexión de la articulación de la rodilla

\section{Fundamentación teórica}

Se flexiona la pierna sobre la cara posterior del muslo, es la única forma natural de flexión de la rodilla y, si a pesar de la tensión muscular que distingue al karateka entrenado, es capaz de cerrar debidamente ambos segmentos corporales, se evidencia un estado óptimo de la capsula articular, los ligamentos implicados y un nivel adecuado de viscosidad y elasticidad muscular, parámetros que influyen directamente en la prevención de lesiones del sistema osteomioarticular.

\section{Metodología}

El karateca se acuesta sobre el tatami en posición de cubito prono y flexiona la pierna sobre la cara posterior del muslo sin ayuda externa.

El profesor debe utilizar un goniómetro para medir la amplitud angular entre ambos segmentos corporales, teniendo en cuenta que mientras más cerrado es este, mejor flexibilidad presenta el atleta.

Procedimiento para medir la amplitud angular de la rodilla con el Goniómetro

- Se deberá estabilizar el segmento proximal

- De cúbito prono el atleta se colocará el fulcro o broche del goniómetro en el centro de la articulación (cóndilo femoral externo), alineando el brazo fijo a la línea media longitudinal del muslo y el brazo fijo se alinea a la línea media longitudinal de la pierna

- Solo se moverá el brazo móvil del goniómetro, manteniendo el fulcro y el brazo fijo del goniómetro en el muslo $\left(0^{\circ}\right.$ grado en posición inicial)

- Lectura del resultado de la medición para la flexión de la articulación de la rodilla ante un movimiento pasivo con presión.

Medios e instrumentos

1. Protocolo de recogida de datos 
2. Goniómetro: Permite evaluar el arco de una articulación; se refiere a la medición de ángulos; ángulos que describen los huesos corporales en sus uniones a la altura de las articulaciones (medir la amplitud angular en la flexión de la rodilla en ambas piernas).

\section{Forma de calificación}

Se le otorga peso mediante una escala Likert a la cual se le asigna un dominio numérico para favorecer el análisis estadístico de los datos, para ello se consideran estudios previos donde se fundamenta una escala de evaluación de la amplitud angular en la flexión de la articulación de la rodilla, la cual oscila entre $0^{\circ} \sin$ flexión y flexionada en un máximo que puede oscilar entre $130^{\circ}$ y $135^{\circ}$ y (Mazza, 2011; Toboadela, 2011: López, 2014).

Criterios para la evaluación de la amplitud angular en la flexión de la rodilla

- Excelente: $x>150^{\circ}$

- Muy Bien: $140^{\circ}<\mathrm{X}$ d» $150^{\circ}$

- Bien: $130^{\circ}<X d » 140^{\circ}$

- Regular: $120^{\circ}<\mathrm{X}$ d» $130^{\circ}$

- Mal: Xd» $120^{\circ}$

En la medición intervendrán dos entrenadores, uno para medir y otro para consignar el valor de cada atleta

\section{Nombre de la Prueba 2: Fuerza muscular}

Objetivo: Comprobar la fuerza muscular asociada a los pateos y desplazamientos que comprometen la articulación de la rodilla

\section{Fundamentación teórica}

Al brindar información sobre la posibilidad del sistema nervioso para el reclutamiento de fibras durante el esfuerzo, es posible inferir el estado de los sistemas neuromusculares de aquellos grupos vinculados a la articulación de la rodilla.

\section{Metodología}

En el banco de cuádriceps se utiliza la máquina para medir el máximo peso que es capaz de levantar el atleta.

Debe ejecutarse una repetición cada vez mientras aumenta progresivamente la resistencia aplicando peso, hasta que el ejecutante no pueda levantar más, entonces se toma el valor del último peso levantado correctamente.

El mismo procedimiento se sigue con el Curl de piernas acostado (bíceps femoral) y con la extensión de tobillos sentado con sobre peso.

\section{Medios e instrumentos}

1. Banco de cuádriceps

2. Protocolo de recogida de datos (Ver anexo 2)

\section{Forma de calificación}

Se consignará el último valor del peso levantado en cada prueba

- Cuádriceps: peso levantado en kilogramos

- Curl de pierna acostado: peso levantado en kilogra-

mos

- Extensión de tobillos sentado

Se saca la media entre los pesos máximos obtenidos para asegurar un valor integral del parámetro

\section{Orientaciones metodológicas generales}

- La relación entre los ejercicios dentro de cada grupo es progresiva, ese mismo modo de relación debe tener lugar entre los grupos de ejercicios, lo que evidencia que el diseño y la selección de los mismos se fundamenta en el principio de sistematización y profundidad del contenido.

- Se respetará la flexibilidad como propiedad del sistema y su adaptabilidad al contexto con el que se relaciona, ya que no solo se puede sino que se deben alternar e intercalar en el proceso con arreglo a los objetivos de cada una las sesiones de trabajo.

- El criterio de progresión de la dificultad y complejidad con vistas al aumento de las cargas va desde las flexiones frontales a las laterales y de estas a las torsiones o rotaciones internas y externas.

- Por último se recomienda que después de las sesiones de trabajo, se apliquen ejercicios de estiramientos para estimular la propiedad esponjosa de los músculos implicados y ejercicios de esfuerzos compensatorios para acelerar el proceso de recuperación por una parte y por la otra, para lograr un desarrollo equilibrado de la fuerza.

\section{Resultado de la comparación de los resultados}

Considerando el análisis sistémico estructural funcional de las acciones del karate en las que se encuentra implicada la articulación de la rodilla durante la ejecución de la kata y el kumité, se realizó un pre-experimento pedagógico el cual sigue la siguiente lógica: medición de la amplitud angular que se deriva de la flexión de la articulación de la rodilla, de la fuerza máxima de los músculos que inervan la articulación de la rodilla y que intervienen en los pateos y los desplazamientos para la kata y el kumité, en un segundo momento se realiza una intervención pedagógica para preparar a los profesores en relación a la aplicación del sistema de ejercicio propuesto, seguidamente se pone en práctica la propuesta durante tres meses, en un segundo momento y bajo las mismas condiciones que el primero se realiza una evaluación.

El análisis general de la validación empírica tiene como punto de partida el registro de la presencia de cuatro (4) atletas exlesionados en la articulación de la rodilla en la población objeto de estudio, la confirmación de las lesiones se derivó de la encuesta implementada en la fase diagnóstica y del análisis del respaldo médico de los atletas que aludían la presencia de la lesión en la articulación de la rodilla, por tanto de manera general la implementación de la propuesta persiguió reducir al máximo la presencia de las lesiones en la articulación de la rodilla como resultado de las exigencias físicas asociadas a la preparación tanto de kata como de kumité en la dinámica de la preparación.

Para el procesamiento de los datos cuantitativos para cada Test se aplicó la prueba de normalidad de KolmogorovSmirnov: KS, los resultados arrojados demostraron que los datos siguen una distribución normal por tanto se cumple el supuesto para la aplicación de la prueba T para diferencias de medias.

\section{Análisis de la contrastación de los datos de la prueba de Goniometría para el pie izquierdo}

La primera prueba que se implementa es la goniometría de la articulación de la rodilla, medición que permitió identificar la amplitud angular que se deriva de la flexión de esta articulación para ambos pies, cuya medición se consignó en grados, se estableció una escala Likert de evaluación cualitativa que osciló entre Excelente y Mal a la que se le dio peso 
en un intervalo numérico que fue desde un (1) punto (Mal) hasta cinco (5) puntos (Excelente).

En el Pre_test, la evaluación de la goniometría en los 14 atletas arrojó que la amplitud angular de la flexión de la articulación de la rodilla osciló en un rango que fue desde los $122^{\circ}$ a los $139^{\circ}$ en el pie izquierdo con una media de $131^{\circ}$ y una desviación típica de 4.5, las evaluaciones se presentaron entre Bien y Regular, este último con nueve casos lo que implica el 64.28\% de la población evaluada.

En el Post_test, posterior a la intervención (ejercicios preventivos) y como resultados del fortalecimiento de los músculos que se inervan la articulación estudiada se apreció que el rango de la amplitud angular de la flexión de la articulación de la rodilla en el pie izquierdo se incrementó en $5^{\circ}$ en relación al valor máximo de la primera medición, los datos consignados oscilaron entre $125^{\circ}$ a $144^{\circ}$ con una media de $136^{\circ}$ y una desviación típica de 5.6 (Tabla 4), lo que indica que los valores que se presentaron de forma más heterogénea en relación al valor central, sin embargo nótese que se evidencian cambio importantes entre ambas mediciones.

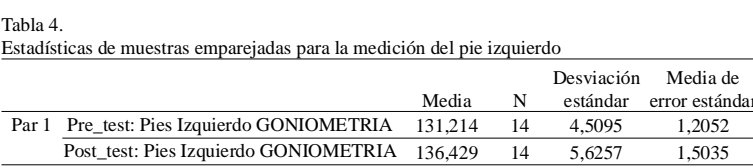

Obsérvese que el promedio en el post test es significativamente superior al promedio en el pre_test en correspondencia a lo planteado.

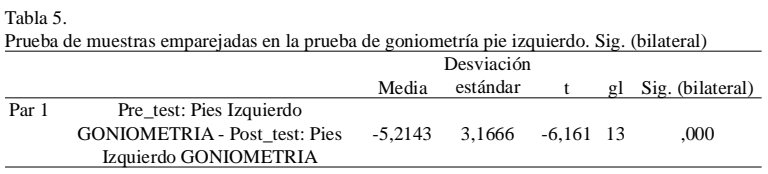

La tabla antes mostrada permite plantear que existen diferencias significativas $(p=.000)$ entre el pre_test y el post_test ya que la significación de la prueba es menor a .05, valor prefijado, lo cual permite corroborar la hipótesis estadística que expone $\mathrm{H} 1$ para la prueba de Goniometría en el pie izquierdo y se rechaza $\mathrm{H}_{0}$. En este orden se reconoce el valor de la propuesta para la prevención de las lesiones de la articulación de la rodilla.

Análisis de la contrastación de los datos de la prueba de Goniometría para el pie derecho

Por su parte los valores en el pre_test en el pie derecho oscilaron entre los $125^{\circ}$ y los $139^{\circ}$ con una media de $131^{\circ}$ y una desviación típica de 4.1, el balance cualitativo se movió para ambos pies entre Regular y Muy Bien, con una preponderancia de la evaluación de Bien con ocho (8) casos para un 57.14\% de la población.

En el post_test los resultados presentados oscilaron entre los $128^{\circ}$ y los $145^{\circ}$ mostrando una diferencia de $6^{\circ}$ en relación al valor máximo consignado, con una desviación típica de 5.2 en relación al pre_test mostrándose los datos menos homogéneos que en el primer momento, los criterios de evaluación se concentraron en el criterio de Bien con ocho (8) casos para un 57.14\% y Muy Bien con cinco (5) casos para un $35.71 \%$, en tal sentido la amplitud articular mejoró de forma significativa al estar el 92.85\% de la población por encima de Bien.

Tabla 6.

Estadísticas de muestras emparejadas para la medición del pie derecho

\begin{tabular}{cccccc}
\hline & & & Desviación & Media de error \\
& & Media & N & estándar & estándar \\
\hline \multirow{2}{*}{ Par 1} & Pre_test: Pies derecho GONIOMETRIA & 131,357 & 14 & 4,1437 & 1,1075 \\
\cline { 2 - 6 } & Post_test: Pies derecho GONIOMETRIA & 136,500 & 14 & 5,2733 & 1,4093 \\
\hline
\end{tabular}

El análisis antes expuesto demuestra una evidente diferencia en los cambios provocados entre el pre_test y el post_test como resultado de la intervención, tal y como se evidencia en la tabla 7 donde $\mathrm{p}=.000$.

Tabla 7.

Prueba de muestras emparejadas en la prueba de goniometría pie derecho. Sig. (bilateral)

\begin{tabular}{cccccc}
\hline & $\begin{array}{c}\text { Media } \\
\text { Desviación } \\
\text { estándar }\end{array}$ & t & gl & Sig. (bilateral) \\
\hline $\begin{array}{c}\text { Par 1 Pre_test: Pies Izquierdo GONIOMETRIA } \\
\text { Post_test: Pies Izquierdo GONIOMETRIA }\end{array} 5,1429$ & 2,8785 & $-6,685$ & 13 &, 000 \\
\hline
\end{tabular}

En correspondencia se acepta la $\mathrm{H}_{1}$ para la prueba de Goniometría en el pie derecho y se rechaza $\mathrm{H}_{0}$ lo que demuestra el valor del sistema de ejercicio como medio preventivo.

Análisis de los resultados de la Prueba de Fuerza Máxima para los cuádriceps

La segunda prueba realizada fue de fuerza máxima del cuádriceps, donde los resultados del pre_test por atleta oscilaron entre los 55 y $75 \mathrm{~kg}$ de peso, con una media $66 \mathrm{~kg}$ y una desviación típica de 5.7, el valor que más se repitió fue de $70 \mathrm{~kg}$ con cinco (5) casos para 31.71\%.

Posterior a la intervención los resultados se mostraron entre 60 y $75 \mathrm{~kg}$, mejorando en (5kg) el valor mínimo, la media se mostró en 69 kg, con una desviación típica de 5.8 .

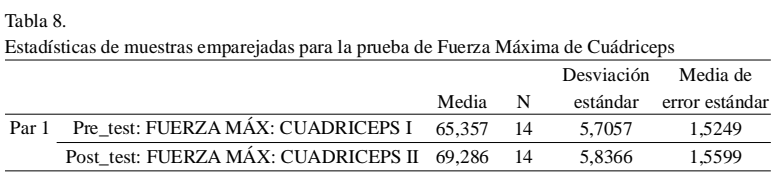

La contratación de los datos manifiestan una diferencia significativa entre el pre y post tratamiento, lo que justifica el valor de $\mathrm{p}=.000$ para la prueba, en concordancia al ser la significación menor que .05 se comprueba $\mathrm{H}_{1}$ y se rechaza $\mathrm{H}_{0}$ para la evaluación de la fuerza máxima del cuádriceps.

Tabla 9.

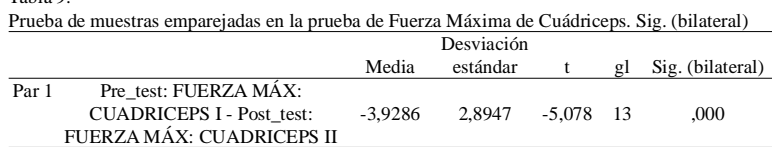

Los antes expuesto lo que demuestra que el fortalecimiento de los músculos que inervan la rodilla previene la aparición de lesiones en la dinámica de la preparación tal y como se consigna en la tabla 9.

Análisis de los resultados de la Prueba de Fuerza Máxima para el Bíceps Femoral

La tercera prueba ejecutada fue la evaluación de la fuerza máxima del Bíceps Femoral, en este orden los resultados del pre_test oscilaron entre los 55 y $75 \mathrm{~kg}$ de peso, con una media $65 \mathrm{~kg}$ y una desviación típica de 5.8, lo que es denotativo que los datos se mostraron un poco disperso alrededor de la 
media, el valor que más se repitió fue de 65 kg con seis (6) casos para un $45.85 \%$.

Posterior a la implementación del sistema de ejercicio los resultados se mostraron entre 60 y $75 \mathrm{~kg}$, mejorando el cinco (5 kg) el valor mínimo del pre_test, la media se mostró en 69 kg, con una desviación típica menor que en el primer momento de 4.9, los resultados se concentraron en $65 \mathrm{~kg}$ con seis (6) casos para un $45.85 \%$ seguido de $75 \mathrm{~kg}$ con custro (4) casos.

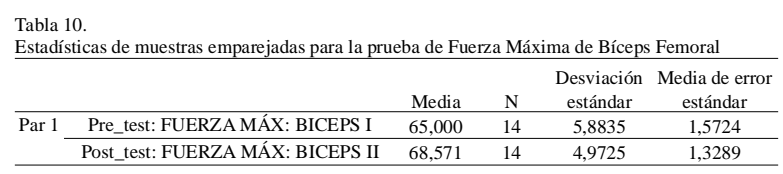

La contratación de los datos muestra una clara diferencia en los cambios provocados entre ambos momentos como resultado de la intervención, de este modo $\mathrm{p}=.000$, al ser menor que .05 , se verifica $\mathrm{H}_{1}$ y se rechaza $\mathrm{H}_{0}$ para la prueba de fuerza máxima de Bíceps Femoral

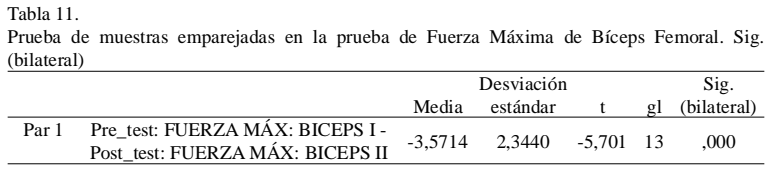

Se corrobora que la fuerza máxima del Bíceps Femoral mejoró de un momento a otro de la evaluación lo que indica que

\section{Análisis de los resultados de la Prueba de Fuerza Máxi- ma para los Gemelos}

La última prueba implementada fue de fuerza máxima en los gemelos, en el primer momento la fuerza máxima de los 14 evaluados osciló entre 220 y $280 \mathrm{~kg}$ de peso, con una media 263 kg y una desviación típica de 16.52, el valor que más se repitió fue de $260 \mathrm{~kg}$ con cuatro (4) casos para un $28.57 \%$ seguido del valor máximo presentado (280 kg) con tres (3) casos para un $21.42 \%$, de manera general los resultados se mostraron heterogéneos en relación al valor medio consignado.

En el segundo momento de la evaluación de la variable se observó una diferencia significativa en relación a los resultados del pre_test. De esta manera los datos oscilaron entre los 240 y $285 \mathrm{~kg}$, mejorando el valor mínimo en $20 \mathrm{~kg}$ y valor máximo en $5 \mathrm{~kg}$ en relación al pre tratamiento.

En concordancia la media se mostró en 265 kg con una desviación típica menor que en el pre_test (11.74), lo que demuestra que los datos se encontraron más concentrados en relación a la media central como se observa en la tabla 12.

Tabla 12.
\begin{tabular}{l} 
Estadísticas de muestras emparejadas para la prueba de Fuerza Máxima de los Gemelos \\
\hline
\end{tabular}
\begin{tabular}{lccccc} 
Media & N & $\begin{array}{r}\text { Desviación } \\
\text { estándar }\end{array}$ & $\begin{array}{c}\text { Media de error } \\
\text { estándar }\end{array}$ \\
\hline Par 1 Pre_test: FUERZA MÁX: GEMELOS & 260,000 & 14 & 16,5250 & 4,4165 \\
\cline { 2 - 6 } & Post_test: FUERZA MÁX: GEMELOS & 265,714 & 14 & 11,7436 & 3,1386 \\
\hline
\end{tabular}

De forma general la contratación de los datos develaron una diferencia significativa entre ambos momentos como resultado de la aplicación del sistema de ejercicios preventivos centrados en el fortalecimiento de los planos que se inervan en la articulación de la rodilla, de esta forma al ser $\mathrm{p}=.003$, al ser menor que .05 como valor prefijado se acepta $\mathrm{H}_{1}$ y se rechaza $\mathrm{H}_{0}$ para la prueba de fuerza máxima de gemelos.

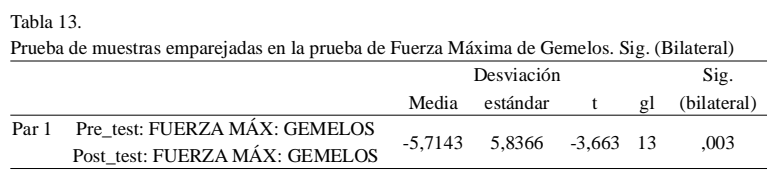

\section{Conclusiones de la fase de validación empírica}

De manera general la fase de validación empírica inicia con cuatro atletas con riesgo de reaparición de lesiones a nivel de la articulación de la rodilla, se culmina el pre-experimento sin la presencia de lesiones y con una mejora significativamente superior en relación al funcionamiento de la articulación como resultado de la implementación de los ejercicios preventivos. Se pudo corroborar que la articulación de la rodilla mejoró la amplitud angular en la flexión, en la población estudiada; se comprueba que los músculos que inervan la articulación de la rodilla mostraron cambios sustanciales en el orden cuantitativo del pre_test al post_test los que contribuyó al fortalecimiento de la capsula articular y a la no aparición de lesiones.

La contrastación de los resultados generales por prueba aplica, resultó determinante para la verificación de la hipótesis de investigación, estos muestran una significación bilateral de .001, al ser menor que el valor prefijado se rechaza la hipótesis nula $\left(\mathrm{H}_{0}\right)$ y se comprueba la hipótesis de investigación $\left(\mathrm{H}_{\mathrm{i}}\right)$.

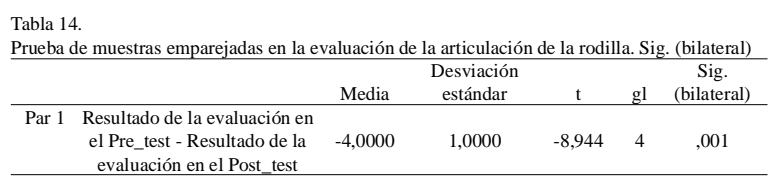

En virtud de lo expresado podemos plantear que «Un sistema de ejercicios específicos elaborados a partir del principio de estructura y función, garantiza la prevención de lesiones de rodillas asociadas las exigencias físicas que se derivan de la ejecución de la kata y el kumité en atletas juveniles de la categoría 14-16 años de la Federación Deportiva de los Ríos».

\section{Discusión de los resultados}

Los resultados expuestos demuestran la necesidad de trabajar en función de la prevención de aquellos planos musculares que tienen una incidencia determinante en el desempeño del atleta, en tal sentido los medios de evaluación empleados aportaron los datos necesarios para orientar la prevención de las lesiones en la articulación de la rodilla en la disciplina objeto de análisis.

Diferentes investigaciones (Sánchez \& Gómez, 2008; Cos, Cos, Buenaventura, Pruna \& Ekstrand, 2010; Feria, et al., 2014b; Martínez-Llobregat, Fernández García \& López, 2014; Latorre, Cámara \& Pantoja, 2014; Romero-Moraleda, Cuéllar, González, Bastida, Echarri, Gallardo \& Paredes, 2017) coinciden en la pertinencia de la prevención de las lesiones como resultado de la aplicación de instrumentos de medición asociados al estado de la fuerza en la articulación estudiada.

En este punto cabe destacar el estudio realizado por Martínez \& Fernández (2016), los autores comparar los valores de potencia y trabajo obtenidos en tiempo real mediante 
los sistemas BlueBrainTMy SmartCoachTM, durante ejercicios de fuerza en una prensa de piernas, equipada con tecnología inercial flywheel, usando diferentes inercias (cargas). Demuestran mediante estas mediciones que en el terreno de la rehabilitación y prevención de lesiones, el realizar este tipo de mediciones permite dosificar de forma más segura y precisa la carga de entrenamiento, ya que no es necesario el uso de cargas máximas para evaluar la fuerza, reduciendo con ello pérdidas, los resultados arrojados coinciden con los expuestos en la presente investigación demostrando la pertinencia y valor del sistema de ejercicio y la dinámica de la intervención asumida en la disciplina estudiada.

Similar estudio realiza Fort \& Romero (2013), los cuales análisis los factores de riesgo neuromusculares de las lesiones deportivas, destacan que dentro de estas se enuncian como las más recurrentes la fatiga muscular, la alteración de la magnitud y los tiempos de activación muscular, la alteración de la capacidad de coactivación muscular, la estrategia de control de la extremidad inferior predominante en el plano frontal, los desequilibrios neuromusculares entre pierna dominante y no dominante, la inadecuada rigidez muscular, los déficits en el control postural, la disminución de la propiocepción, los déficits de núcleo y la disminución en los mecanismos de anticipación. Como resultado proponen una guía práctica para el diseño de programas de prevención para cada tipo de deporte, demostrando la pertinencia del instrumento para la problemática presentada.

Cabeza-Carmona, Barranco-Ruiz \& Villa-González (2019) proponen un programa de intervención orientado a la prevención de lesiones, demuestran mediante su aplicación en la práctica que este mejoró de forma significativa la movilidad de la articulación proclive a la lesión por su nivel de implicación en la actividad competitiva.

Por otra parte Penichet-Tomás et al (2019), realizan un estudio mediante el cual develan las lesiones más frecuentes en jugadores españoles de élite de balonmano playa, como resultado demuestran el patrón lesivo más recurrente, los datos aportados por los autores indican que el $28.7 \%$ de los deportistas sufrían dolor crónico, siendo la causa del 26.3\% la vieja lesión.

Los autores apuntan que los resultado del estudio constituyen un referente para favorecer la prevención de lesiones, agregan que las nuevas investigaciones relacionadas deberían estudiar también la etiología de las lesiones crónicas ya que estas pueden afectar tanto a la salud como al rendimiento de los deportista, posicionamiento que le otorga valor al presente estudio y a los resultado que se derivan del mismo (Penichet-Tomás, et al., 2019).

\section{Conclusiones}

La teoría que respalda la prevención de lesiones de rodilla en los deportistas, los objetivos y contenidos de la actividad física profiláctica, las métodos y medios que se emplean para su proyección, el análisis biomecánico de los movimientos con repercusión directa en la articulación de la rodilla así como las alternativas preventivas para la disciplina de Karate Do, permitieron arribar a una caracterización integral y detallada del estado actual del objeto de estudio.

El diagnóstico realizado al proceso de preparación física de los grupos musculares comprometidos con las funciones de la rodilla de los karatekas de la categoría juvenil de 14-16 años de Federíos develan que los criterios de dosificación de las cargas presentan un carácter progresivo lineal y no obedecen a los principios del entrenamiento deportivo y se demuestra una falta de conocimiento sobre la selección y el uso de los medios específicos y auxiliarles.

El diagnóstico reveló múltiples irregularidades que justifican la objetividad del problema científico declarado y la necesidad de establecer alternativas viables que preserven la calidad del desempeño de los karatecas de la categoría 1416 años de la Federación Deportiva de los Ríos a partir de la profilaxis ante la aparición de lesiones de rodilla asociadas a las exigencias físicas que se derivan de la ejecución de la kata y el kumité, lo cual devela incumplimiento del principio profiláctico morfo-fisiológico para el entrenamiento deportivo según Agüero, Gómez, Quesada, Nelson \& Aquino (2015).

El sistema de ejercicios específicos está en correspondencia con los resultados del diagnóstico y por tanto se alinea a la prevención de las lesiones asociadas a las exigencias físicas que se derivan de la ejecución de la kata y el kumité.

El sistema de ejercicios diseñado, se caracteriza por su carácter sostenible, generalizable y progresivo, articula estructuralmente una entrada, un proceso y una salida que permite retroalimentarse a través de su sistema de evaluación.

La validación teórica de la propuesta develó una alta coincidencia en relación a la calidad formal, importancia y utilidad del sistema de ejercicios para la prevención de lesiones, la evaluación de la estructura develó criterios positivos en relación al sistema de objetivos, la fundamentación teórica que respalda la propuesta, el sistema de principios que fundamenta la propuesta, su representación gráfica y sistema de evaluación.

La evaluación empírica del sistema de ejercicios mostró cambios significativos entre el pre tratamiento y el post tratamiento, lo que permitió comprobar la hipótesis de investigación planteada para la situación experimental.

\section{Referencias}

Adalid, L. J. (2014). Propuesta de incorporación de tareas preventivas basadas en métodos propioceptivos en fútbol. Retos. Nuevas tendencias en Educación Física, Deporte y Recreación, 163-167.

Agüero, O. J., Gómez, A. B., Quesada, J. G., Nelson, D. A., \& Aquino, L. R. (2015). Principio de profilaxis morfo-fisiológica para el entrenamiento deportivo. Realidades y perspectivas. Efdeportes, 1-3.

Álvarez, J., \& Murillo, V. (2016). Evolución de la prevención de lesiones en el control del entrenamiento. Arch Med Deporte, 37-58.

Ayala-Mejías, J. D., García-Estrada, G. A., \& Alcocer, P. E. (2014). Lesiones del ligamento cruzado anterior. Acta ortop. mex, 2-9.

Cabeza-Carmona, M. J., Barranco-Ruiz, Y., \& Villa-González, E. (2019). Programa de prevención de lesiones para la mejora de la salud articular del hombro en jóvenes triatletas. Retos, 35, 80-86 
Cos, F., Cos, M. A., Buenaventura, L., Pruna, R., \& Ekstrand, J. (2010). Modelos de análisis para la prevención de lesiones en el deporte. Estudio epidemiológico de lesiones: el modelo Union of European Football Associations en el fútbol. Apunts. Medicina de l'Esport (45)166. 57148

De Armas, R., Lorences, G. J., \& Perdomo, V., J. M. (2001). Caracterización y diseño de los resultados científicos como aportes de la investigación educativa. Universidad Pedagógica «Félix Varela». La Habana

Feria, M.A., Fernández, R. K., Romero, B. S., Cortés, J. M., \& Sañudo, C. B. (2014a). Diferencias de género en la estabilización de rodilla en aterrizajes de salto. Retos. Nuevas tendencias en Educación Física, Deporte y Recreación, 178-179.

Feria, M. A., De Hoyo, L. M., Romero, B. S., Mateo, C. J., \& Sañudo, C. B. (2014b). Varo y valgo de rodilla en cambios de dirección como factor de riesgo de lesión. Retos. Nuevas tendencias en Educación Física, Deporte y Recreación, 176-177.

Fernández, M., \& Busto, J. M. (2015). Prevención de lesiones deportivas. Medigraphic, 94-105.

Fernández, N. B. (2014). Lesiones mas frecuentes en el Karate. Federación Argentina de Karate Do y Kobudo Okinawense (FAKKO).

Francesc, M., Buenaventurac, M. A., Prunad, R., \& Ekstrande, J. (2016). Modelos de análisis para la prevención de lesiones en el deporte. Estudio epidemiológico de lesiones: el modelo Union of European Football Associations en el fútbol. Apunts. Medicina de l'Esport, 95-102.

Fort, V.A., \& Romero, R. D. (2013). Análisis de los factores de riesgo neuromusculares de las lesiones deportivas factores de riesgo neuromuscular de lesiones deportivas. Apunts. Medicina de l'Esport.(48), 179.

De Dios, D. F. (2016). Las lesiones en el karate modalidad de Kumite (combate). V Torneo internacional de Kárate, $1-18$.

García, O. T. (2012). Prevención de lesiones del ligamento cruzado anterior. IV Congreso Internacional de Ciencias del Deporte y la Educación Física, (2-7).

Latorre, R. P., Cámara, P. J., \& Pantoja, V.A. (2014). Efectos de un programa de educación para la seguridad en el deporte en escolares de secundaria. Retos. Nuevas tendencias en Educación Física, Deporte y Recreación, 5-8.

López-Liria, R., Vega-Ramirez, F.A., López-Liria, R., CatalánMatamoros, D., Padilla-Góngora, D., Martínez-Cortés, M. C., \& Mesa-Ruiz, A. (2012). La rehabilitación y fisioterapia domiciliaria en las prótesis de rodilla. An Sist Sanit Navar, 99-113.

López, G. L. F. (2014). Goniometría. Resumen visual de Goniometria. 1a ed. - Buenos Aires : Asociart ART, Extraido desde: https://es.slideshare.net/fher1810/resumen-visual-de-goniometria?qid

Martínez-Llobregat, A. J., Fernández García, E. M., \& López, E. J. (2014). Prevención de lesiones en la realización de deporte. Lesiones más frecuentes. Aldadis.net La revista de educación, 25-28.

Martínez,A. L. M., \& Fernández, G. R. (2016). Comparación de dos dispositivos de medición de potencia y trabajo durante ejercicio de fuerza con tecnología inercial flywheel. Retos, (29), 144-148

Mazza, J. C. (2011). Métodos de evaluación de la amplitud del movimiento mediante métodos goniométricos. Consultado 23 de octubre, Recuperado desde https:// docplayer.es/421878-Metodos-de-evaluacion-de-la-amplitud-del-movimiento-mediante-metodosgoniometricos.html

Mundo Deportivo. (4 de Agosto de 2016). Mundo deportivo. Obtenido de http://www.mundodeportivo.com/juegos-olimpicos/20160804/403675110027/antonio-espinoses-un-paso-trascendente-para-el-karate.html

Penichet-Tomás, A., Ortega, B. M., Jiménez-Olmedo, J. M., Pueo, B., \& Espina, A. J. J. (2019). Incidencia lesiva en jugadores españoles de élite de balonmano playa. Retos, 36, 83-86.

Pitarch, D. (2016). Medicina Deportiva. Lesiones en el Karate. Web. Karate y Algo más. Consultado el 5 de septiembre del 2018. Extraído desde: https:// karateyalgomas.com/2015/07/18/medicina-deportiva-lesiones-frecuentes-en-karate/

Prieto, A. J. M. (2015). Variables deportivas y personales en la ocurrencia de lesiones deportivas. Diferencias entre deportes individuales y colectivos. Retos, 28, 21-25.

Romero-Moraleda, B., Cuéllar, A., González, J., Bastida, N., Echarri, E., Gallardo, J., \& Paredes, V. (2017). Revisión de los factores de riesgo y los programas de prevención de la lesión del ligamento cruzado anterior en fútbol femenino: propuesta de prevención. RICYDE. Revista internacional de ciencias del deporte, 117-138.

Sánchez, M. B., Rodríguez-Mansilla, J., \& González, S. B. (2015). Recuperación de la artroplastia de rodilla a través de la movilización pasiva continua. An. Sist. Sanit. Navar, 297-310.

Sánchez, J. F., \& Gómez, C. A. (2008). Epidemiología de las lesiones deportivas en baloncesto. Revista Internacional de Medicina y Ciencias de la Actividad Física y el Deporte. (8)32, 270-281

Toboadela, C. (2011). Goniometría. Una herramienta para la medición de las incapacidades laborales. 1a.ed. - Buenos Aires. Asociart, ART. Extraido desde https:// www.slideshare.net/santinozenteno/goniometria72923042

Villaquirán, A. F., Portilla, E., \& Vernaza-Pinzón, P. (2016). Caracterización de la lesión deportiva en atletas caucanos con proyección a Juegos Deportivos Nacionales. Rev Univ. Salud, 541-549.

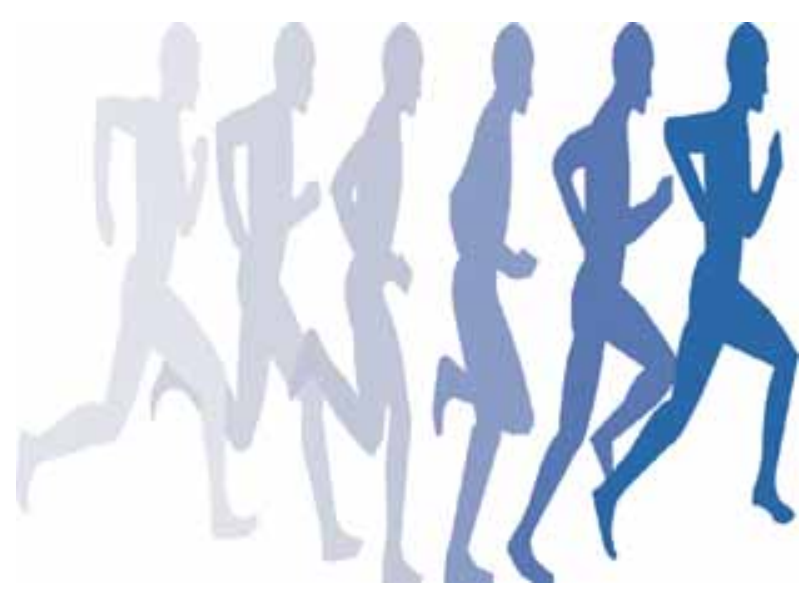

